\title{
Percepção de trabalhadores sobre indicadores como ferramenta para gestão da qualidade em hematologia
}

Workers 'perception of indicators as a tool for hematology quality management

Percepción de los trabajadores sobre los indicadores como herramienta para la gestión de la calidad de la hematología

Karen Saboia Aragão e Silva

ORCID: https://orcid.org/0000-0002-0452-6646 Universidade Federal do Rio Grande do Norte, Brasil E-mail: karenaragao@gmail.com

Eliane Santos Cavalcante

ORCID: https://orcid.org/0000-0002-0001-9161 Universidade Federal do Rio Grande do Norte, Brasil E-mail: elianeufrn@hotmail.com

Lianne Clarissa Cavalcanti Eufrázio de Araújo ORCID: https://orcid.org/0000-0003-0201-1451 Universidade Federal do Rio Grande do Norte, Brasil E-mail: lianneclarissa@ufrn.edu,br

Éricles Ferreira Sales de Oliveira

ORCID: https://orcid.org/0000-0001-5310-8486 Universidade Federal do Rio Grande do Norte, Brasil

E-mail: ericles.ferreira3@outlook.com

Thaiza Teixeira Xavier Nobre

ORCID: https://orcid.org/0000-0002-8673-0009 Universidade Federal do Rio Grande do Norte, Brasil E-mail: thaizax@hotmail.com

Micheline Veras de Moura

ORCID: https://orcid.org/0000-0001-8519-637X Hospital Universitário Onofre Lopes, Brasil

E-mail: michelineverasenf@yahoo.com

Viviane Peixoto dos Santos Pennafort

ORCID: https://orcid.org/0000-0002-5187-4766

Hospital Universitário Onofre Lopes, Brasil Universidade Federal do Rio Grande do Norte, Brasil E-mail: vivipspf@yahoo.com.br

Rayra Mass Lucena de Sena Lima ORCID: https://orcid.org/0000-0002-9408-6401 Universidade Federal do Rio Grande do Norte, Brasil E-mail: rayra.mass@hotmail.com Verbena Santos Araújo ORCID: https://orcid.org/0000-0002-3519-4744 Universidade Federal do Rio Grande do Norte, Brasil E-mail: verbena@ufrn.edu.br

Elisangela Guerra de Sousa

ORCID: https://orcid.org/0000-0001-7411-3241 Universidade Federal do Ceará, Brasil E-mail: elisguerrabrito@ hotmail.com

\section{Resumo}

Objetivou-se compreender a percepção de uma equipe de colaboradores sobre a aplicabilidade do Sistema INDICAH como ferramenta de gestão de qualidade em um centro de hematologia de um município do nordeste brasileiro. Tratase de estudo qualitativo e descritivo realizado com participação de 23 trabalhadores lotados na instituição de realização do estudo, o qual ocorreu no período de fevereiro a abril de 2020. Para a coleta dos dados, utilizou-se da técnica grupo focal. Extraiu-se duas categorias das narrativas: Estratégias para melhoria do sistema de indicadores de qualidade e Estratégias para acesso aos indicadores de forma fácil. A interpretação das categorias ocorreu mediante análise temática de Bardin. As falas gravadas nos grupos focais foram transcritas para documento Word, em seguida, foram inseridas no software Atlas-ti, versão 8.4.24.0, que favoreceu a codificação dos dados e a organização em categorias e subcategorias. $\mathrm{O}$ estudo permitiu compreender a percepção da equipe de trabalhadores em relação à 
aplicabilidade do Sistema INDICAH como ferramenta de gestão de qualidade no HEMOCE. Estes achados contribuem para o planejamento de estratégias para melhoria da gestão de qualidade por meio do uso de indicadores, tais como, melhor comunicação, relação setorial, maior divulgação dos indicadores e formas de conquistar o doador moldados de acordo com as necessidades locais.

Palavras-chave: Gestão da qualidade; Avaliação em saúde; Serviço de hemoterapia; Indicadores de qualidade em assistência à saúde.

\begin{abstract}
The objective was to understand the perception of a team of collaborators about the applicability of the INDICAH System as a quality management tool in a hematology center in a municipality in northeastern Brazil. This is a qualitative and descriptive study carried out with the participation of 23 workers assigned to the institution where the study was carried out, which took place from February to April 2020. For data collection, the focus group technique was used. Two categories were extracted from the narratives: Strategies for improving the quality indicator system and Strategies for easily accessing the indicators. The interpretation of the categories occurred through thematic analysis of Bardin. The speeches recorded in the focus groups were transcribed into a Word document, then they were inserted in the Atlas-ti software, version 8.4.24.0, which favored the coding of the data and the organization into categories and subcategories. The study made it possible to understand the perception of the team of workers in relation to the applicability of the INDICAH System as a quality management tool in HEMOCE. These findings contribute to the planning of strategies to improve quality management through the use of indicators, such as better communication, sectorial relations, greater dissemination of indicators and ways to win the donor shaped according to local needs.
\end{abstract}

Keywords: Quality management; Health evaluation; Hemotherapy service; Quality indicators, health care.

\title{
Resumen
}

El objetivo fue conocer la percepción de un equipo de colaboradores sobre la aplicabilidad del Sistema INDICAH como herramienta de gestión de la calidad en un centro de hematología de un municipio del noreste de Brasil. Se trata de un estudio cualitativo y descriptivo realizado con la participación de 23 trabajadores adscritos a la institución donde se realizó el estudio, el cual se llevó a cabo de febrero a abril de 2020. Para la recolección de datos se utilizó la técnica de grupos focales. Se extrajeron dos categorías de las narrativas: Estrategias para mejorar el sistema de indicadores de calidad y Estrategias para acceder fácilmente a los indicadores. La interpretación de las categorías se produjo a través del análisis temático de Bardin. Los discursos registrados en los grupos focales se transcribieron a un documento de Word, luego se insertaron en el software Atlas-ti, versión 8.4.24.0, que favoreció la codificación de los datos y la organización en categorías y subcategorías. El estudio permitió comprender la percepción del equipo de trabajadores en relación a la aplicabilidad del Sistema INDICAH como herramienta de gestión de la calidad en HEMOCE. Estos hallazgos contribuyen a la planificación de estrategias para mejorar la gestión de la calidad a través del uso de indicadores, como mejor comunicación, relaciones sectoriales, mayor difusión de indicadores y formas de ganar al donante conformado de acuerdo a las necesidades locales.

Palabras clave: Gestión de la calidad; Evaluación en salud; Servicio de hemoterapia; Indicadores de calidad de la atención de salud.

\section{Introdução}

Considera-se que a qualidade nas organizações de saúde vem sendo cada vez mais discutida e compartilhada entre os profissionais, com vistas à excelência dos serviços prestados. Nas últimas décadas, os usuários têm se apropriado gradativamente de seus direitos e do exercício da cidadania, o que requer, desse modo, maior comprometimento dos prestadores de serviços (Brasil, 2016).

Nessa direção, a história da hemoterapia no Brasil nas últimas três décadas registrou importantes avanços na busca de um sistema hemoterápico que oferecesse para a população um produto final com segurança e qualidade. Isso só foi possível graças à reestruturação dos serviços, legitimação da doação de sangue como ato voluntário, altruísta e não remunerado, além dos avanços tecnológicos, legislações, normatizações técnicas, capacitações e modernização da gestão. A Hemorrede Pública Brasileira foi seriamente assumindo a missão de garantir o fornecimento de sangue para toda a população de forma segura e sustentável, buscando a seleção de candidatos à doação saudáveis, voluntários e regulares (Brasil, 2015).

Para a avaliação do Sistema de Gestão da Qualidade a análise dos indicadores faz parte dos processos de segurança no uso do sangue. A mentalidade de risco é essencial para se conseguir um sistema de gestão da qualidade eficaz. A abordagem de 
riscos e oportunidades estabelece uma base para o aumento da eficácia do sistema de gestão da qualidade, conseguir resultados melhorados e para a prevenção de efeitos negativos (Abnt, 2015). Os indicadores são ferramentas utilizadas para a organização monitorar determinados processos quanto ao alcance ou não de uma meta ou padrão mínimo de desempenho estabelecido.

O padrão de desempenho esperado no uso de indicadores de qualidade necessita de avaliação contínua a fim de qualificar e promover melhoramento, considerando ser um processo contínuo. Desse modo, além da fundamentação do estudo, deve-se levar em consideração a qualidade da pesquisa, e isso exige dos profissionais envolvidos na análise um conhecimento aprofundado sobre os diferentes tipos de estudos e quais são adequados para atender a temática com melhor qualidade.

Em relação aos tipos de estudo temos quatro desenhos de pesquisa clínica: ensaio clínico, estudos de coorte, estudos caso-controle e transversal. Cada um desses tipos de pesquisa pode responder de alguma forma mais específica ao contexto estudado e ser utilizado como base para aprofundamento na construção de protocolos (Pimenta, Pastana, Sichieri, Solha \& Souza, 2015).

Os indicadores de qualidade podem ser uma estratégia de mensurar e avaliar as ações de enfermagem. Considerados instrumentos de gestão que orientam o caminho para a excelência do cuidado, eles se constituem na maneira pela qual os profissionais de saúde verificam uma atividade, monitoram aspectos relacionados a determinada realidade e avaliam o que acontece com os pacientes, apontando a eficiência e eficácia de processos e os resultados organizacionais (Januário, Lemos, Friche \& Alves, 2015; Gabriel et al 2011).

Visando alcançar importantes níveis de qualidade, produtividade, competitividade, controle dos processos e segurança no ambiente de trabalho, tem- se cada vez mais optado pela padronização dos processos, por acreditar-se que essa é uma ferramenta importante na administração organizacional das funções assistenciais (Coldebella \& Segalla, 2019).

O estudo apresenta grande relevância científica, situando-se em um campo teórico e epistemológico de prevenção, ao gerar visibilidades pela necessidade de enxergar no serviço de hematologia e hemoterapia, o impacto dos indicadores de qualidade na assistência à Saúde, contribuindo para a prevenção de risco e de eventuais não conformidades, assim como, na promoção da saúde.

Diante dessas considerações, objetivou-se compreender a percepção de uma equipe de colaboradores sobre a aplicabilidade do Sistema INDICAH como ferramenta de gestão de qualidade em um centro de hematologia de um município do nordeste brasileiro.

\section{Metodologia}

Trata-se de um estudo exploratório-descritivo na abordagem qualitativa. Nesta perspectiva, considera-se que os métodos qualitativos requerem a interpretação por parte dos pesquisadores por meio de suas opiniões e reflexões acerca do fenômeno em estudo (Pereira et al, 2018).

Os participantes do estudo foram trabalhadores que atuam no Hemocentro Regional de Sobral, sendo em sua maioria, gestores de área que assumem a coordenação de um setor do serviço. Neste estudo participaram dez enfermeiros, seis farmacêuticos bioquímicos, dois técnicos de enfermagem, dois técnicos de laboratório, um técnico em hemoterapia e dois profissionais técnicos da área administrativa, totalizando 23 trabalhadores lotados na instituição do estudo, no período de fevereiro a abril de 2020 .

Como critérios de inclusão, foram utilizados a atuação por, no mínimo, um ano na instituição e o requisito de compor o grupo de estudo sobre indicadores de qualidade. Foram excluídos os profissionais afastados por motivo de férias, licençamaternidade e licença médica no período da coleta de dados. Assim, aceitaram fazer parte da pesquisa, assinando o Termo de Consentimento Livre e Esclarecido (TCLE), 23 colaboradores. 
Para a coleta dos dados, utilizou-se a técnica do grupo focal. Foram realizados três grupos focais, com duração média de 60 minutos cada. A coleta de informações foi registrada através de aparelho eletrônico $\mathrm{mp} 4$ mediante a autorização escrita dos participantes. A análise dos dados foi realizada conforme a técnica de análise de conteúdo temática de Bardin adaptada por Minayo (Minayo, 2010).

Os dados foram submetidos à análise de conteúdo na modalidade temática. Inicialmente, realizou-se a fase exploratória de investigação. Depois, foi realizado o momento interpretativo, no qual se utilizou dos relatos dos participantes para dar sentido, lógica e projeção às respostas, buscando sua compreensão e agregação. Na sequência, realizou-se a ordenação dos dados que englobam a transcrição do material obtido por meio das entrevistas, do grupo focal e da releitura do material. Em seguida, iniciou-se a classificação dos dados por meio da leitura horizontal e exaustiva dos textos, permitindo apreender as estruturas de relevância e as ideias centrais. Na leitura transversal, foram separados os dados por unidades de sentido, ou seja, temas. No processo classificatório, identificaram-se as categorias, unindo as partes semelhantes, buscando perceber as conexões entre elas e guardando-as em códigos (Minayo, 2010).

As falas gravadas nos grupos focais foram transcritas para documento Word, em seguida, foram inseridas no software Atlas-ti, versão 8.4.24.0, que favoreceu a codificação dos dados e organização em categorias e subcategorias, por ser um suporte tecnológico para sistematização da análise e o tratamento dos dados.

Todavia, vale ressaltar que todas as inferências e categorizações devem ser feitas pelo pesquisador, considerando o seu conhecimento da literatura sobre o tema em questão. O software Atlas-ti contribui para a codificação dos dados, a otimização do processo analítico, além de ser maleável para inúmeros tipos de pesquisas qualitativas com objetivos diversos (Silva Júnior \& Rattner, 2016).

Esse estudo seguiu os preceitos éticos sendo aprovado pelo CEP- HUOL/UFRN com Número do Parecer 3.676.720 de 01 novembro de 2019 e CAAE: 23017119.1.0000.5292.

\section{Resultados e Discussão}

Quanto ao perfil sociodemográfico dos participantes, grande parte tinha acima de 40 anos (69,50\%); 73,90\% eram do sexo feminino e o mesmo percentual caracterizou-se na cor parda; 78,20\% eram casados, com maior prevalência da religião católica (87,0\%); 91,30\% informaram ter graduação com, ou sem pós-graduação; todos eram procedentes da cidade de Sobral CE. Em relação ao cargo ocupado, os gestores de área foram maioria com 60,80\%. O tempo de atuação no hemocentro ultrapassou os dez anos em 60,90\% dos casos; 56,60\% não possuíam outro vínculo de trabalho. Quando indagados acerca da capacitação em gestão da qualidade, todos afirmaram que regularmente recebiam algum treinamento relacionado à temática.

O tempo de atuação na instituição e as capacitações periódicas são fatores capazes de mitigar inconformidades e otimizar a qualidade do serviço. Alguns autores ressaltam que a implementação de treinamento teórico-prático acerca das complicações relacionadas às não conformidades, assim como, a atualização de documentos e o desempenho de uma equipe de coleta habilitada, influencia sobremaneira na redução do descarte de sangue total. (Coutinho, Bello, Hammer \& Hammer, 2020).

A partir da leitura, organização e subsequente classificação dos dados, emergiram duas categorias: Estratégias para melhoria do sistema de indicadores de qualidade e Estratégias para acesso aos indicadores de forma fácil.

\section{Estratégias para melhoria do sistema de indicadores de qualidade}

A primeira categoria apresenta o reconhecimento das participantes acerca dos indicadores de qualidade. Nas entrevistas e discussões do grupo focal, os indicadores de qualidade foram apontados como elementos mensurados 
rotineiramente, que evidenciam o resultado do trabalho assistencial e possibilitam identificar se os objetivos do atendimento estão sendo atingidos. Alguns depoimentos demonstram esse entendimento:

"Os indicadores são úteis pois determinamos os índices de perdas, gastos e qualidade do produto a ser enviado ao destinatário final” [G1]

"Então se a gente pudesse ter como melhoria, essa estratégia da ferramenta de divulgação maior através das redes sociais, até mensagem de whatsapp facilitaria muito o nosso trabalho de captação, porque o nosso trabalho é um trabalho de formiguinha, porque a gente precisa conscientizar o doador, a gente precisa esclarecer a importância da doação de sangue e todas as ferramentas que a gente puder usar pra conseguir esse objetivo, é melhor".'[G2]

A fim de assegurar a conscientização do doador de sangue é importante a existência de indicadores bem definidos quanto ao gerenciamento de riscos. Os indicadores desenvolvidos e validados em um estudo realizado por Gama et al (2016) em três hospitais brasileiros contribuíram para a gestão da segurança do paciente, apresentando instrumentos aprovados quanto à validade, confiabilidade, viabilidade e utilidade para o gerenciamento dos riscos assistenciais.

Recomenda-se ainda, a presença de medidas para diminuição do risco de danos, com potencial para incorporação nas políticas de saúde seguras fundadas em evidência (Covo, 2018).

Ainda foi mencionado pelos participantes que o indicador de qualidade é uma ferramenta de melhoria do cuidado prestado ao público usuário do serviço de hematologia e que, além de auxiliar a gestão no conhecimento do doador de sangue, a fim de fidelizá-lo para evitar perdas de doações, pode ainda auxiliar na tomada de decisão clínica no que tange às boas práticas no atendimento, conforme relatado a seguir:

"Quem está lá no processamento ou quem está lá na investigação da qualidade desse sangue, vai ter o mesmo compromisso que eu tenho: conquistar, fidelizar. Tem uma frase que é a cara de um captador que diz que captar é uma história bonita de fazer, mas dificil de viver. Às vezes a gente consegue tudo, mas quando chega na instituição, a gente perde esse doador porque ele foi mal recepcionado". [G3]

O relato oriundo do grupo focal (G3) está em consonância com outro estudo, o qual destacou que que a busca pela excelência dos serviços em hemoterapia envolve a identificação correta dos erros existentes no processo como um todo, a fim de que possa ser realizada a implantação de melhorias em todas as etapas do processo. Os itens a serem avaliados incluem a verificação das condições da estrutura física da agência transfusional, do controle da qualidade de equipamentos e insumos utilizados nos procedimentos hemoterápicos e a proposição de ações e medidas corretivas para as situações que possam afetar a Garantia de Qualidade (Stein et al, 2017)

Percebe-se que os depoimentos fornecidos nas falas do grupo focal convergem quanto ao reconhecimento da importância dos indicadores, o que reforça seu conceito como ferramenta de gestão para avaliar a qualidade da assistência no centro de hematologia estudado e, se necessário, modificá-la conforme as evidências, voltadas para as boas práticas na gestão do cuidado. A prática baseada em evidências é ferramenta essencial para o resultado dos pacientes; assim, o enfermeiro, ao buscar melhorias fundamentadas para a clínica deste, contribui com os resultados gerenciais (Lamont, Brunero, Lyons, Foster \& Perry, 2015).

Outrossim, o processo de avaliação não deve se constituir isoladamente e restrito ao esforço de um profissional, mas sim, ter característica de prática institucionalizada e com o envolvimento dos diversos atores da instituição. Recomenda-se que os ciclos de avaliação sejam formativos, processuais e contínuos, não se esgotando na resolução da não conformidade identificada, visto que a avaliação é somente parte de um processo dinâmico dos serviços e que, exige gerenciamento permanente dos processos de trabalho (Brasil, 2016). 
De maneira geral, infere-se que o acompanhamento sistemático dos indicadores de qualidade é primordial para prevenir, não conformidades no processo de trabalho da unidade estudada. O HEMOCE utiliza o modelo proposto pelo Ministério da Saúde (Brasil, 2015) que norteia o processo de implantação dos indicadores em todas as áreas do ciclo do sangue, visando obter uma análise mais concreta da situação do serviço para então propor ações mais efetivas. Dada as peculiaridades do ambiente das unidades de hemoterapia, especificamente no HEMOCE por configurar rede de apoio às demais unidades do Ceará, apresenta-se o contexto a seguir nas falas dos colaboradores para compreensão da situaçãoproblema que desafiou seus gestores.

Nesse contexto, o gestor tem papel fundamental na tomada de decisões de forma eficaz, norteada pela prática baseada em evidências e não somente planejada e organizada empiricamente a partir de demandas cotidianas.

Figura 1 - Distribuição quanto às estratégias para melhoria do sistema de indicadores de qualidade descritos pela equipe de trabalhadores do centro de hematologia. Sobral-CE, 2020.

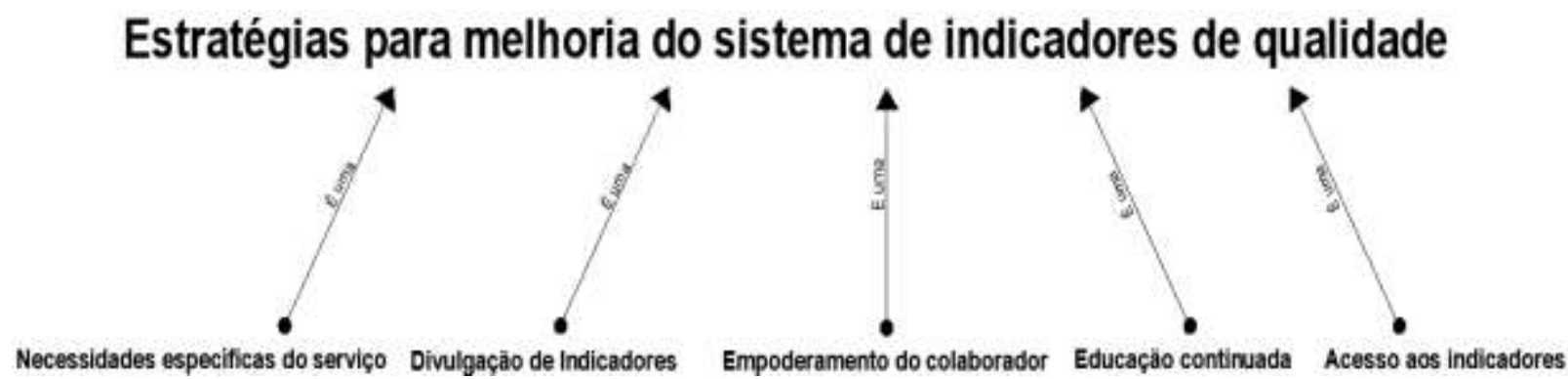

Fonte: Dados da pesquisa (2020).

$\mathrm{Na}$ Figura 1 observou-se as principais estratégias para melhoria do sistema de indicadores elencadas a partir dos relatos emergidos dos grupos focais. Segundo consolidado das falas, os indicadores de qualidade são uma necessidade do serviço, que devem ser divulgados e incentivados, a fim de empoderar o colaborador por meio de educação continuada com facilidade de acesso aos indicadores.

Nesta perspectiva, pesquisadores corroboram ao enfatizar que apesar dos avanços observados ao longo dos anos na hemoterapia brasileira, ainda persistem riscos potenciais que precisam ser analisados e tratados para a adequação dos serviços (Silva Júnior \& Rattner, 2016). Dessa forma, a utilização de indicadores como ferramenta de gestão da qualidade nos serviços de hemoterapia, proporciona uma avaliação acurada das metas a serem alcançadas por meio das ações realizadas, no direcionamento da melhoria da assistência.

\section{Estratégias para acesso aos indicadores de forma fácil}

Essa categoria abordou como os trabalhadores vêm utilizando os indicadores de qualidade no contexto do HEMOCE de forma a facilitar o acesso. A maioria dos participantes trabalham com os indicadores de maneira retrospectiva, pois, no final de cada mês, há uma análise crítica dos resultados em que é traçado um plano de ação com a equipe de colaboradores e a gestão para adequar os indicadores que não atingiram a meta proposta. 
Figura 2 - Distribuição quanto à utilidade dos indicadores na prática descritos pela equipe de trabalhadores do centro de hematologia. Sobral-CE, 2020.

\section{Utilidade dos indicadores da prática}

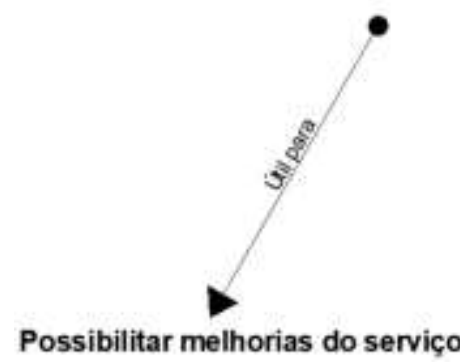

Possibilitar melhorias do serviço
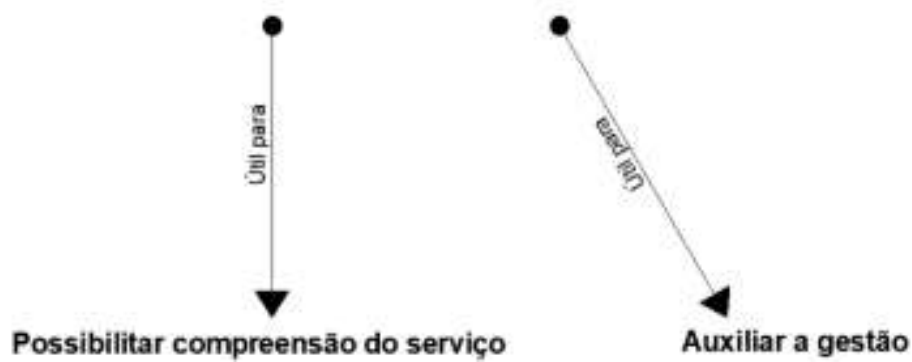

Fonte: Dados da pesquisa (2020).

A Figura 2 destaca a relevância dos indicadores no contexto da hematologia conforme percepção dos trabalhadores, com ênfase na compreensão das demandas dos serviços e nas oportunidades de melhorias identificadas viabilizadas por meio das ações da gestão da qualidade.

Importante destacar que a análise periódica do indicador em questão permite que, prontamente, sejam estabelecidos planos de ação para corrigir as "não conformidades" caso ocorram e adequá-las às metas planejadas, conferindo dinamicidade ao processo.

Essa segunda categoria de análise pode ser ilustrada com estes depoimentos:

"Então eu acho que para melhorar a gente precisaria aperfeiçoar a descentralização das informações que são colocadas ali, que as pessoas tivessem acesso a esses indicadores e que elas se implicassem cada vez mais com o que está sendo colocado ali, com essa realidade"[G3]

"Fazer um informativo, chamar o colaborador para estar junto com o gestor na hora da alimentação do indicador é uma coisa que eu faço muito. Eu me sinto privilegiada em ter uma pessoa que tem o interesse de entender aquilo, de olhar, de ter essa visão crítica, mas em outros setores já não tem”. [G4]

"O nosso sistema de indicadores é muito fácil, acessível. É fácil a alimentação dos dados, do plano de ação, da análise crítica” [G4].

Infere-se com as falas dos entrevistados que os indicadores de qualidade são reconhecidos e aceitos como ferramenta indispensável para o gerenciamento de boas práticas no ambiente HEMOCE. Os relatos analisados demonstram o envolvimento e a disposição da equipe no sentido de avaliar se as metas assistenciais foram atingidas e otimizar o cuidado ao usuário, bem como prevê melhorias na dinâmica do trabalho centrado nesse usuário, auxiliando no planejamento e na tomada de decisão coparticipativa usuário-equipe-serviço para a prática baseada em evidências.

Entende-se por evidências científicas as informações cientificamente fundamentadas que justificam as ações propostas. Desse modo, no estudo avaliado, o profissional deve associar o seu conhecimento adquirido à sua experiência. Num estudo com forte evidência científica deve-se incluir: a descrição das estratégias de buscas da evidência (revisão da literatura), a força da recomendação, a identificação do percentual da recomendação, considerações sobre segurança das ações, práticas não efetivas e mau uso potencial em diferentes cenários e localização, custo-efetividade e escassez de recursos (Brasil, 2018). 
Acrescenta-se que a prática baseada em evidências tem sido implementada na prática clínica e indicada objetivando a promoção da segurança e da qualidade dos cuidados de saúde. (Bandeira, Lapão \& Madruga; 2017)

Nesse contexto a fim de assegurar a prática de segurança nos centros de hematologia, a Portaria MS/GM 158 (Brasil, 2016) regulamenta os procedimentos adotados pelos hemocentros, recomendando que na avaliação da adequação do local e da estrutura, se verifique a compatibilidade entre a equipe e o número de doadores esperado e a presença de, pelo menos, um médico e um enfermeiro para a constituição da equipe de coleta externa. Essa Portaria aponta ainda como indicador de qualidade a necessidade de verificação da compatibilidade da infraestrutura com o processo de coleta, a fim de permitir fluxo adequado, iluminação e ventilação da área física para coleta de sangue, privacidade para a triagem clínica, condições adequadas para o atendimento de intercorrências clínicas dos doadores e condições para oferta de hidratação oral e descanso após a doação (Covo, 2018).

Acredita-se que o uso de ferramentas tende a aprimorar a assistência, favorecer o uso de práticas cientificamente sustentadas, minimizar a multiplicidade das informações e condutas entre os membros da equipe de saúde, estabelecer limites de ação e cooperação entre os diversos profissionais, além de serem instrumentos construídos dentro dos princípios da prática baseada em evidências que oferecerem as melhores condutas de cuidado (Pimenta et al, 2015).

Os indicadores nesse sentido funcionam como uma ferramenta/protocolo guia para direcionamento eficaz do cuidado seguro com previsão de riscos aos usuários/cliente/paciente. Assim, com o intuito de definir uma situação específica da assistência ou de um cuidado prestado, os protocolos assistenciais têm o objetivo de descrever detalhadamente as ações operacionais e especificações sobre o modo de execução dessas, bem como, orientar o profissional executor. Esses instrumentos podem facilitar a redução da variabilidade de conduta entre os diferentes profissionais envolvidos na assistência à saúde, favorecer maior segurança para o paciente, permitir elaboração de indicadores de processos e resultados, aprimorar a qualidade da assistência, bem como, o uso racional de recursos (Lemos, Poveda, \& Peniche, 2017).

Para uma melhor compreensão dos profissionais quanto ao uso dos protocolos assistenciais, estes devem ter suas condutas descritas e preconizadas de forma clara e precisa quanto aos resultados esperados, além de revisados periodicamente, considerando a realidade local, instituição em que está sendo aplicada, como também, atualizações que possam ocorrer no âmbito dos novos estudos mundiais. A construção de protocolos deve ser baseada em evidências científicas, fundamentados nos elementos de qualidade, quantidade e consistência dos estudos revisados (Lemos, Poveda \& Peniche, 2017).

Dessa forma, observa-se a necessidade de preparo minucioso com participação ativa de toda a equipe de colaboradores desde a seleção dos indicadores e a elaboração dos protocolos, com o propósito de identificar e analisar os resultados, com ênfase no uso racional do sangue nos serviços de hemoterapia.

As principais dificuldades e limitações encontradas no decorrer do estudo relacionaram-se com a dinâmica dos setores e turno de trabalho dos profissionais envolvidos, tornando a reunião de um grupo focal no horário do trabalho algo difícil. A realização dos grupos focais não foi possível fora do horário de trabalho, pois alguns trabalhadores estavam com carga horária diferenciada e/ou com outros vínculos empregatícios.

\section{Conclusão}

Este estudo possibilitou analisar as falas dos trabalhadores de um Centro de Hematologia do Nordeste brasileiro, quanto a utilização e as contribuições dos indicadores de qualidade nas práticas gerenciais no âmbito local. Nesse contexto, evidenciou-se que os indicadores de qualidade são reconhecidos pelos colaboradores como ferramentas de mensuração da qualidade da assistência prestada ao doador de sangue e ao paciente hematológico desse serviço de hemoterapia.

Quanto à utilização do sistema INDICAH, vêm sendo abordados pela equipe de trabalhadores do HEMOCE mediante discussões, a construção de sua análise crítica e de seus planos de ação feita pela equipe gerencial por meio da educação 
Research, Society and Development, v. 10, n. 2, e8310212203, 2021

(CC BY 4.0) | ISSN 2525-3409 | DOI: http://dx.doi.org/10.33448/rsd-v10i2.12203

continuada em serviço. A díade ensino-serviço a partir das demandas dos trabalhadores favorecendo o processo de excelência no cuidado ao usuário atendido no serviço revelando o alcance dos objetivos propostos, bem como auxiliando no processo de melhoria contínua do cuidado e da tomada de decisão acerca da prevenção de eventos de não conformidades, favorecendo as boas práticas de cuidado.

Ressalta-se a necessidade de outras investigações acerca da utilização do sistema de indicadores como ferramenta de gestão da qualidade nos serviços de hematologia, a fim de avaliar contextos diversos na identificação e validação de estratégias eficazes para a melhoria da qualidade da assistência à saúde na hemoterapia.

\section{Referências}

Associação brasileira de normas técnicas. NBR ISO 9001:2015: Sistemas de gestão da qualidade - Requisitos, 2015.

Bandeira, A. G., Witt, R. R., Lapão, L. V., \& Madruga, J. G. (2017). A utilização de um referencial metodoló gico na implementação de evidências como parte da investigação em enfermagem. Texto \& Contexto-Enfermagem, 26(4).

Coldebella, V., \& Segalla, P. (2019). O Procedimento Operacional Padrão no Serviço Aeromédico do Oeste do Paraná. News Resgates Aeromédicos. https://www.resgateaeromedico.com.br/wp-content/uploads/2019/01/ARTIGO-P\%C3\%93S-AEROM\%C3\%89DICO-VANESSA.pdf

Coutinho, C. M., Bello, C. M., Hamer, \& Hamer, E. R. (2020). Complicatedness associated to whole blood discard in the Hemotherapy Service in the National Institute of Cancer. $R B A C, 52(1), 35-41$.

Covo, M. Z. (2018). Matriz de recomendações para melhoria de desempenho do ciclo do sangue no Hemocentro Coordenador do Estado do Paraná. https://www.prppg.ufpr.br/siga/visitante/trabalhoConclusaoWS?idpessoal=30251\&idprograma=40001016073P0\&anobase=2018\&idtc=26.

Gabriel, C. S., Melo, M. R. A. D. C., Rocha, F. L. R., Bernardes, A., Miguelaci, T., \& Silva, M. D. L. P. (2011). Use of performance indicators in the nursing service of a public hospital. Revista latino-americana de enfermagem, 19(5), 1247-1254.

Gama, Z. A. D. S., Saturno-Hernández, P. J., Ribeiro, D. N. C., Freitas, M. R. D., Medeiros, P. J. D., Batista, A. M., \& Melo Neto, V. D. (2016). Desenvolvimento e validação de indicadores de boas práticas de segurança do paciente: Projeto ISEP-Brasil. Cadernos de Saúde Pública, 32 , e0026215.

Januário, G. C., Lemos, S. M. A., Friche, A. A. D. L., \& Alves, C. R. L. (2015). Quality indicators in a newborn hearing screening service. Brazilian journal of otorhinolaryngology, 81(3), 255-263.

Lamont, S., Brunero, S., Lyons, S., Foster, K., \& Perry, L. (2015). Colaboração entre equipes de liderança de enfermagem clínica: um estudo sequencial explicativo de métodos mistos. J Nurs Manag, 23(8), 1126-36.

Lemos, C. D. S., Poveda, V. D. B., \& Peniche, A. D. C. G. (2017). Construction and validation of a nursing care protocol in anesthesia. Revista latinoamericana de enfermagem, 25.

Minayo, M. C. D. S. (2010). The challenge of knowledge: qualitative research in health: Hucitec.

Ministério da Saúde. (2015). Secretaria de Atenção à Saúde. Departamento de atenção hospitalar de Urgência. Coordenação geral de sangue e hemoderivados. Guia para implantação do programa Nacional de Qualificação Hemorrede: Ministério da Saúde.

Ministério da Saúde. (2016). Portaria ${ }^{\circ}$ 158, de 04 de fevereiro de 2016. Redefine o regulamento técnico de procedimentos hemoterápicos. Diário Oficial da União, Brasília, 2016. https://www.in.gov.br/web/dou/-/portaria-n-158-de-4-de-fevereiro-de-2016-22301274.

Ministério da Saúde. (2016). Secretaria de Atenção à Saúde. Departamento de Atenção Especializada e Temática. Guia para implementar avaliações nos serviços de hematologia e hemoterapia na perspectiva do Programa Nacional de Qualificação Hemorrede: Ministério da Saúde, 2016. http://bvsms.saude.gov.br/bvs/publicacoes/guia_implementar_avaliacoes_servicos_hematologia.pdf

Pereira A. S. et al. (2018). Metodologia da pesquisa científica. UAB/NTE/UFSM. https://repositorio.ufsm.br/bitstream/handle/1/15824/Lic_C omputacao_Metodologia-Pesquisa-Cientifica.pdf?sequence=1

Pimenta, C. A. D. M., Pastana, I. C. A., Sichieri, K., Solha, R. K., \& Souza, W. (2015). Guia para construção de protocolos assistenciais de enfermagem. In Guia para construção de protocolos assistenciais de enfermagem.

Silva Júnior, J. B., \& Rattner, D. (2016). A Vigilância Sanitária no controle de riscos potenciais em serviços de hemoterapia no Brasil. Saúde em Debate, 40, 136-153.

Stein, B. D. P., Imeton, T. S., Geraldo, A., Bueno, E. C., Stringari, F. B., \& Martinello, F. (2017). Avaliação da Gestão da Qualidade de uma Agência Transfusional. Rev. bras. ciênc. saúde, 203-210. 